\title{
Multicenter phase II study of brequinar sodium in patients with advanced lung cancer*
}

\author{
Jean Maroun ${ }^{1}$, John Ruckdeschel2***, Ronald Natale ${ }^{3}$, Robert Morgan ${ }^{4}$, Brian Dallaire $^{5}$, Regina Sisk ${ }^{5}$, \\ John Gyves 5 \\ 1 Ottawa Regional Cancer Center, Ottawa, Canada \\ 2 Albany Medical Center, Albany, NY, USA \\ ${ }^{3}$ University of Michigan, Ann Arbor, MI, USA \\ ${ }^{4}$ City of Hope National Medical Center, Duarte, CA, USA \\ 5 The DuPont Pharmaceutical Co., Wilmington, DE, USA
}

Received 24 April 1992/Accepted 10 November 1992

Summary. A total of 53 patients with advanced lung cancer [non-small-cell (NSC), 21; small-cell (SC), 32] were treated with brequinar sodium. All of the NSC patients were chemotherapy-naive, but $31 / 32$ (97\%) SC patients had failed a multiagent chemotherapy program prior to study entry. Brequinar was given intravenously at a median weekly dose of $1200 \mathrm{mg} / \mathrm{m}^{2}$. The toxicity was moderate, with 19 patients $(36 \%)$ experiencing grade 3 or 4 toxicity. Objective responses were observed in one NSC and two SC patients. We conclude that at this dose and on this schedule, brequinar does not have sufficient activity in patients with NSC or in patients with previously treated SC to warrant further evaluation. However, since responses were observed in previously treated SC lung-cancer patients, further evaluation in chemotherapy-naive patients may be warranted.

\section{Introduction}

Brequinar is a novel antimetabolite that interferes with dihydrooratate dehydrogenase, an enzyme crucial for pyrimidine biosynthesis [4]. Brequinar was selected for clinical development because of its broad antitumor activity in murine models (L1210 leukemia and B16 melanoma) and human tumor xenografts [breast (MX-1), colon (CX-1), lung (LX-1), and gastric (BL/STX-1)] [7].

\footnotetext{
* This study was supported by The DuPont Pharmaceutical Co., Wilmington, Delaware
}

** Current affiliation: H. Lee Moffitt Cancer Center, Tampa, FL

Correspondence to: J. W. Gyves, Medical Director, Oncology, The DuPont Pharmaceutical Company, P. O. Box 80026 Wilmington, DE 19880-0026, USA
A number of phase I studies using different dose levels and schedules have been reported $[1-3,5,8,9]$. In these phase I studies, the dose-limiting toxicities included thrombocytopenia and mucositis/stomatitis. On the basis of the phase I experience, the recommended phase II starting dose of brequinar on the weekly intravenous schedule was $1,800 \mathrm{mg} / \mathrm{m}^{2}[2,3]$. However, early in the phase II evaluation of brequinar in several tumor categories, this starting dose resulted in unacceptable toxicity [6]. As a result, the starting dose was reduced to $1,200 \mathrm{mg} / \mathrm{m}^{2}$ weekly. This multicenter study was designed to evaluate the activity of brequinar in patients with advanced nonsmall-cell (NSC) and small-cell (SC) lung cancer.

\section{Patients and methods}

NSC patients were chemotherapy-naive and SC patients had received at most one prior chemotherapy regimen. Additional criteria for inclusion. in the study were a performance status of $\leq 2$ (WHO scale), a life expectancy of $>8$ weeks, a serum bilirubin level of $<1.5 \mathrm{mg} / \mathrm{dl}$, a serum creatinine value of $<2.0 \mathrm{mg} / \mathrm{dl}$, an absolute granulocyte count of $>1,500$ cells $/ \mathrm{mm}^{3}$, a platelet count of $>100,000 / \mathrm{mm}^{3}$, and the presence of bidimensionally measurable disease. Informed consent was given by all patients in accordance with regulatory agency requirements.

Brequinar was given intravenously once weekly at a starting dose of 1,800 ( 3 patients) or $1,200 \mathrm{mg} / \mathrm{m}^{2}$ ( 50 patients) in $500 \mathrm{ml}$ normal saline over $1-2 \mathrm{~h}$. The subsequent dose of brequinar was escalated or decreased by a fixed amount as specified in the protocol according to predetermined criteria and depending on the toxicities experienced in the preceding course. When necessary, dosing was delayed until the patient had recovered from toxicities. Toxicity was coded by NCI common toxicity criteria ( $2 / 18 / 88$ version).

Patients were interviewed and examined prior to each dose of chemotherapy. Laboratory studies including complete blood cell, differential, and platelet counts were repeated once a week. Total protein, albumin, calcium, inorganic phosphorous, glucose, blood urea nitrogen, creatinine, uric acid, total bilirubin, alkaline phosphatase, lactic dehydrogenase (LDH), and electrolytes were assessed every 4 weeks.

Response was evaluated every 4 weeks by appropriate radiology studies and clinical measurement of bidimensional lesions. Criteria for defining response were standard except that a palpable reduction in liver size was not used to designate a partial response [10]. Patients were considered to be evaluable for response if they received at least one dose of brequinar and underwent a subsequent assessment of their measurable 
Table 1. Patient and treatment characteristics

\begin{tabular}{lcc}
\hline Characteristics & NSC & SC \\
\hline Total patients & 21 & 32 \\
(inevaluable/not eligible) & $(3 / 2)$ & $(1 / 0)$ \\
Men: women & $17 / 4$ & $17 / 15$ \\
Median age (range) & $60(39-77)$ & $63(41-77)$ \\
& years & years \\
Performance status ${ }^{\mathrm{a}}$ : & & \\
0 & 2 & 10 \\
1 & 14 & 19 \\
2 & 5 & 3 \\
Sites of metastases: & & \\
Liver & $3(14 \%)$ & $15(47 \%)$ \\
Lung & $13(61 \%)$ & $18(56 \%)$ \\
Lymph nodes & $5(24 \%)$ & $8(25 \%)$ \\
Bone & $4(19 \%)$ & $5(16 \%)$ \\
Skin/subcutaneous tissue & $0(0)$ & $4(12 \%)$ \\
Other & $5(24 \%)$ & $9(28 \%)$ \\
Prior therapy: & & 14 \\
Surgery/diagnostic procedure & 11 & 17 \\
Radiation therapy & 6 & 31 \\
Biological therapy & 0 & \\
Chemotherapy & 0 & \\
Baseline LDH: & & \\
Mean/median & $245 / 166 \pi / 1$ & \\
\hline
\end{tabular}

a World Health Organization criteria [10]

Table 2. Dosing and treatment results

\begin{tabular}{lcc}
\hline & NSC & SC \\
\hline Doses given: & & \\
$\quad$ Total & 152 & 191 \\
Median & 7 & 4 \\
$\quad$ Range & $1-24$ & $1-20$ \\
Median weekly dose & $1,200 \mathrm{mg} / \mathrm{m}^{2}$ & $1,200 \mathrm{mg} / \mathrm{m}^{2}$ \\
$\quad$ Range) & $\left(768-2,986 \mathrm{mg} / \mathrm{m}^{2}\right)$ & $\left(740-1,500 \mathrm{mg} / \mathrm{m}^{2}\right)$ \\
& & \\
Number of patients: & & \\
$\quad$ Dose escalation & 8 & 3 \\
Dose reduction & 6 & 11 \\
Receiving one dose only & 1 & 2 \\
Treatment results: & & \\
$\quad$ PR & 1 & 9 \\
Stable disease & 8 & \\
Progressive disease & 23 & \\
\hline
\end{tabular}

disease. Patients who died of disease without undergoing such an assessment were considered to be evaluable and classified as having progressive disease.

\section{Results}

From January 1989 to July 1990, 53 patients with histologically or cytologically documented, advanced, measurable carcinoma of the lung were entered in the study.
Table 3. Toxicity encountered ${ }^{a}$

\begin{tabular}{lcccl}
\hline Toxicity & \multicolumn{3}{c}{ Number of patients by maximal grade } \\
\cline { 2 - 5 } & Grade 1 & Grade 2 & Grade 3 & Grade 4 \\
\hline Anorexia & 3 & 1 & 0 & 0 \\
Fatigue & 5 & 2 & 1 & 0 \\
Nausea/vomiting & 22 & 6 & 4 & 0 \\
Mucositis/stomatitis & 14 & 9 & 2 & 0 \\
Rash & 3 & 5 & 1 & 0 \\
Diarrhea & 13 & 6 & 6 & 0 \\
Thrombocytopenia & 9 & 9 & 3 & 7 \\
Anemia & 1 & 14 & 6 & 3 \\
Leukopenia & 12 & 4 & 1 & 2 \\
Granulocytopenia & 5 & 9 & 2 & 1 \\
\hline
\end{tabular}

a All patients were evaluable for toxicity

Patient and treatment characteristics are listed in Table 1. The patients had excellent performance status. Of the 32 SC patients, 31 had previously received multiagent chemotherapy; $25(81 \%)$ of these 31 patients had shown a response [partial response (PR), 14; complete response (CR), 11] to prior chemotherapy. Those patients who had achieved a CR to this prior therapy had been off treatment for a median of 167 days (mean, 210 days) prior to the initiation of brequinar treatment. The predominant sites of metastatic disease were the liver, lung, lymph nodes, and bone.

The dosing and treatment results are presented in Table 2. The 53 patients received 343 doses of brequinar (median, 5; range, 1-24). In 11 patients dose escalation was possible, and in 17 patients dose reduction was required; 3 patients received only 1 dose. The median weekly dose was $1,200 \mathrm{mg} / \mathrm{m}^{2}$. Of the 53 patients, 49 were evaluable for response. Two patients were deemed ineligible: one due to the absence of a measurable lesion and the other due to a history of prior malignancy. In addition, two patients were inevaluable due to early non-disease-related death and inability to obtain follow-up tumor measurements. PRs were observed in one NSC and two SC patients.

All 53 patients were evaluable for toxicity. In general, the toxicity encountered in this phase II trial was moderate. The major nonhematologic and hematologic toxicities encountered are listed in Table 3. There was no treatment-related death. In all, 13 episodes of grade 4 toxicity occurred in $8(15 \%)$ patients (NSC, 3; SC, 5). In one NSC patient the grade 4 toxicity was associated with the $1,800-\mathrm{mg} / \mathrm{m}^{2}$ starting dose; the remaining instances of grade 4 toxicity occurred in patients whose starting dose was $1,200 \mathrm{mg} / \mathrm{m}^{2}$ and generally occurred during the early treatment doses. Seven patients experienced grade 4 thrombocytopenia. Five of these patients received platelet transfusions and four showed evidence of bleeding; however, in only two patients was the bleeding sufficient to require replacement. The grade 3 toxicities summarized in Table 3 occurred in $11(21 \%)$ patients (NSC, 8; SC, 3). In two NSC patients who experienced grade 3 toxicity the starting dose was $1,800 \mathrm{mg} / \mathrm{m}^{2}$; the remaining grade 3 toxicities occurred in patients whose starting dose was $1,200 \mathrm{mg} / \mathrm{m}^{2}$. 
Grade 3-4 toxicity was observed in all three patients entered at the initial starting dose of $1,800 \mathrm{mg} / \mathrm{m}^{2}$. The reduction in the starting dose to $1,200 \mathrm{mg} / \mathrm{m}^{2}$ resulted in a more acceptable level of grade 3-4 toxicity (16/50 patients, $32 \%)$. Dose escalation was possible in $21 \%(11 / 53)$ of patients; however, despite the reduction in the starting dose, a further dose reduction was required at some point in the course of treatment in 32\% (17/53) of patients. The reduction in the starting dose followed by additional escalations and reductions based on individual patient tolerance account for the wide range (740$2,986 \mathrm{mg} / \mathrm{m}^{2}$ ) of weekly doses given to these patients with lung cancer. The dose given to SC patients was escalated less frequently than that given to NSC patients, and the SC patients required dose reduction more frequently. This variation in tolerance may in part reflect the greater hepatic involvement in the SC patient group as well as their prior exposure to chemotherapy.

\section{Discussion}

The response rate of $5 \%$ (one-sided $95 \%$ confidence limit, $26 \%$ ) obtained in NSC lung cancer and that of $6 \%$ (onesided $95 \%$ confidence limit, 20\%) obtained in SC lung cancer are disappointing. On the basis of these results, we conclude that at this dose and on this schedule, brequinar sodium does not have sufficient activity to warrant further evaluation in patients with advanced NSC or previously treated SC lung cancer. However, since responses were observed in previously treated SC lung-cancer patients, further evaluation in chemotherapy-naive patients may be warranted.

\section{References}

1. Armand J, Fontana X, DeForni M, Carde P, Munck N, Cvitkovic E, Malet Martino MC (1987) A phase I study of DuP 785 (NSC 368390 ) using a 5 daily IV schedule. Proc Am Soc Clin Oncol 6: 46

2. Arteaga C, Brown T, Kuhn J, Shen H, O'Rourke T, Beougher K, Brentzel H, Von Hoff D, Weiss G (1989) Phase I clinical and pharmacokinetic trial of brequinar sodium (DuP 785, NSC 368390). Cancer Res 49: 4648-4653

3. Bork E, Vest S, Hansen H (1989) A phase I clinical and pharmacokinetic study of brequinar sodium, DuP 785 (NSC 368390), using a weekly and a bi-weekly schedule. Eur J Clin Oncol 25: 1403-1411

4. Chen S, Ruben R, Dexter D (1986) Mechanism of action of the novel anticancer agent 6-fluoro-2-(2'-fluoro-1, 1'-biphenyl-4-yl)-3-methyl4-quinoline carboxylic acid sodium salt (NSC 368 390): inhibition of de novo pyrimidine nucleotide biosynthesis. Cancer Res 46: 50145019

5. Currie V, O'Hehir M, Baltzer L, Slavik W, Yaldaei S, Bertino J (1988) Phase I trial of DuP 785 given on a single weckly intravenous dosing schedule. Proc Am Soc Clin Oncol 7: 76

6. Dallaire B, Varns C, Galbraith D, Wielgosz G, Adams D, Brentzel H, Lynch W, Carlson R, Sisk R, Azarnia N, Bigelow R, Barbu M, Gyves J, Grillo-Lopez A (1991) Preliminary report of safety for a phase II trial of brequinar sodium (DuP 785, NSC 368 390) in refractory solid tumors. Clin Res 39: 375

7. Dexter D, Hesson D, Ardecky R, Rao G, Tippett D, Dusak B, Paull K, Plowman J, DeLarco B, Narayanan V, Forbes M (1985) Activity of a novel 4-quinolinecarboxylic acid, NSC 368390 [6-fluoro-2-(2'fluoro-1,1'-biphenyl-4-yl)-3-methyl-4-quinolinecarboxylic acid sodium salt], against experimental tumors. Cancer Res 45: $5563-$ 5568

8. Noe D, Rowinsky E, Shen H, Clarke B, Grochow L, McGuire W, Hantel A, Adams D, Abeloff M, Ettinger D, Donehower R (1990) Phase I and pharmacokinetic study of brequinar sodium (NSC 368 390). Cancer Res 50: 4595-4599

9. Schwartsmann G, Dodion P, Vermorken J, Bokkel Huinink W ten, Joggi J, Winograd B, Gall H, Simonetti G, Vijgh W van der, Hennik M van, Crespeigne N, Pinedo H (1990) Phase I study of DuP 785 (NSC 368930 ) in solid tumors. Cancer Chemother Pharmacol 25: $345-351$

10. WHO (1979) Handbook for reporting results of cancer treatment. Official publication 45 . WHO, Geneva 